\title{
Polarization-singular Processing of Phase-inhomogeneous Layers Laser Images to Diagnose and Classify their Optical Properties
}

\author{
Yuriy O. USHENKO ${ }^{1}$, Yuriy Ya. TOMKA ${ }^{1}$, Igor Z. MISEVICH ${ }^{1}$, Anton-Pavlo ANGELSKY ${ }^{1}$, \\ Victor T. BACHINSKY ${ }^{2}$ \\ ${ }^{1}$ Chernivtsi National University, 58012, Chernivtsi, Ukraine \\ ${ }^{2}$ Bucovinian State Medical University, 58000, Chernivtsi, Ukraine \\ yuriyu@gmail.com
}

\begin{abstract}
Adduced in this work are the results of investigation aimed at analysis of coordinate distributions for azimuths and ellipticity of polarization (polarization maps) in laser images of three types of phase-inhomogeneous layers (PhIL), namely: rough, ground and bulk scattering layers. To characterize polarization maps for all the types of PhIL, the authors have offered to use three groups of parameters: statistical moments of the first to fourth orders, autocorrelation functions, logarithmic dependences for power spectra related to distributions of azimuths and ellipticity of polarization inherent to PhIL laser images. Ascertained are the criteria for diagnostics and classification of PhIL optical properties.
\end{abstract}

Index Terms-autocorrelation, phase-inhomogeneous layer, polarization, rough surface, statistical moments.

\section{INTRODUCTION}

By tradition, the processes of transforming optical radiation of phase-inhomogeneous objects and media are considered, as a rule, in a statistical approach (theory of radiation transfer [1], Monte-Carlo modeling [2]). Among the most spread traditional methods for studying the scattered light fields, one can separate the following independent directions: "scalar" (photometry and spectrophotometry) [3, 4] and "vector" (polarization nephelometry, Mueller-matrix optics) [5 - 32]. Using these approaches, determined are interrelations between the sets of statistical moments of the 1 -st to 4 -th orders $[6,7,11,15$, $20,26,27,31]$, correlation functions [13, 18, 19, 22, 27], fractal dimensionalities $[6,7,26]$ that characterize phaseinhomogeneous or rough surfaces and coordinate distributions for phases $[15,16,19,28]$, azimuths and ellipticity of polarization in their laser images [31-50].

In parallel with traditional statistical investigations, formed in recent 10 to 15 years is the new optical approach to describe a structure of polarizationally inhomogeneous fields in the case of scattered coherent radiation. The main feature of this approach is the analysis of definite polarization states to determine the whole structure of coordinate distributions for azimuths and ellipticities of polarization. The so-called polarization singularities are commonly used as these states [5, 23, 29]:
* states with linear polarization when the direction of rotation for the electric field vector is indefinite, the socalled L-points;

* circularly-polarized states when the azimuth of polarization for the electric field vector is indefinite, the socalled C-points.

This work is aimed at ascertaining the possibilities to diagnose and classify phase-inhomogeneous layers (PhIL) of various types (surface-scattering, subsurface-scattering and bulk-scattering ones) by determination values and ranges for changing the statistical (moments of the 1-st to 4th orders), correlation (autocorrelation functions) and fractal (logarithmic dependences for power spectra) parameters that characterize coordinate distributions for polarizationsingular states in PhIL laser images.

\section{MAIN MODEL CONCEPTIONS AND ANALYTICAL RELATIONS}

As a base for analytical description of processes providing formation of polarization-inhomogeneous images for various types of PhIL, we have used the model conceptions developed in the works $[5,13-15,16,19,20$, 28]:

- surface-scattering PhIL is a rough surface (superficial layer of the skin epithelium) consisting of an ensemble of quasi-plane, chaotically oriented micro-areas with optical dimensions $l \succ \lambda$ - group 1;

- PhIL with surface and subsurface scattering - ground glass with rough external and subsurface (the layer of collagen fibrils of the skin derma) components - group 2;

- PhIL with bulk scattering - optically thick layer of the skin derma of a various optical thickness - group 3.

\subsection{Mechanisms providing formation of polarization- inhomogeneous images for rough surface}

Optical properties of each micro-area of rough layer of the epithelium are exhaustively characterized with the Jones operator of the following look:

$$
\{R\}=\left\|\begin{array}{cc}
1 & 0 \\
0 & p_{y} / p_{x}
\end{array}\right\| .
$$


It is possible to show that within the sizes $(\Delta x, \Delta y)$ of one micro-area there takes place the change of polarization azimuth $\alpha$ inherent to the refracted plane-polarized laser wave with the initial azimuth $\alpha_{0}$ :

$\alpha(\Delta x, \Delta y)=\operatorname{arctg}\left(p_{y} U_{0 y} / p_{x} U_{0 x}\right)=\operatorname{arctg}\left[\left(\Delta p_{x y}\right) \operatorname{tg} \alpha_{0}\right]$

where $U_{0 x}, U_{0 y}$ are orthogonal components of the amplitude $U_{0}, p_{x}, p_{y}$ - Fresnel amplitude coefficients for transmission [5].

Thus, in the approach of one-fold scattering the polarization image of rough surface may be considered as coordinate-distributed parts of $L$-polarized states [23, 29].

\subsection{Model structure of PhIL with surface and subsurface components - ground surfaces}

The process providing formation of a local polarization state can be considered as superposition of "influences" of an optically strained subsurface of optically anisotropic layer of collagen fibrils as well as the surface rough microrelief one disposed in sequence. From the analytical viewpoint, this scenario can be described by superposition $\{F\}$ of the Jones matrix operators for these partial layers (subsurface $\{T\}$ and surface $\{R\}$ ) :

$\{F\}=\{R\}\{T\}=\left\|\begin{array}{ll}f_{11} & f_{12} \\ f_{21} & f_{22}\end{array}\right\|=\left\|\begin{array}{ll}\left(r_{11} t_{11}+r_{12} t_{21}\right) ; & \left.\left(r_{11} t_{12}+r_{12} t_{22}\right) ;\right) ; \\ \left(r_{21} t_{11}+r_{22} t_{21}\right) ; & \left(r_{21} t_{12}+r_{22} t_{22}\right)\end{array}\right\|$,

$\{T\}=\left\|\begin{array}{ll}t_{11} & t_{12} \\ t_{21} & t_{22}\end{array}\right\|=$

$\left.=\| \begin{array}{cc}\left.\cos ^{2} \gamma+\sin ^{2} \gamma \exp (i \delta)\right] ; & {[\cos \gamma \sin \gamma \exp (i \delta)] ;} \\ {[\cos \gamma \sin \gamma \exp (i \delta)] ;} & {\left[\sin ^{2} \gamma+\cos ^{2} \gamma \exp (i \delta)\right.}\end{array}\right]$

Here, $\gamma$ is the direction of the optical axis of fibril; $\delta$ - phase shift between orthogonal components $\left(U_{x}, U_{y}\right)$ of the amplitude $(U)$ of laser wave with the wavelength $\lambda$ that arises as a consequence of birefringence in the matter $\Delta n$.

If taking into account the relations (1), (3) and (4), it follows that within the limits $(\Delta x, \Delta y)$ of a local bulk formed is an elliptically polarized part of the object field with the following parameters.

$$
\begin{aligned}
& \tilde{\alpha}(\Delta x, \Delta y)= \\
& =\operatorname{arcos}\left(\sin \delta / \cos 2\left(\operatorname{arctg}\left\{\left[\left(f_{21}+f_{22}\right)^{2} /\left(f_{11}+f_{12}\right)^{2}\right] \operatorname{tg} \alpha_{0}\right\}\right)\right)
\end{aligned}
$$

$$
\begin{aligned}
& \tilde{\beta}(\Delta x, \Delta y)= \\
& =\arcsin \left(\operatorname{tg} \delta / \sin 2\left(\operatorname{arctg}\left\{\left[\left(f_{21}+f_{22}\right)^{2} /\left(f_{11}+f_{12}\right)^{2}\right] \operatorname{tg} \alpha_{0}\right\}\right)\right)
\end{aligned}
$$

As it follows from the analytical relations (5) and (6), interaction of the plane-polarized $\left(\alpha_{0}\right)$ wave with the PhIL of this type provides formation of a polarizationinhomogeneous laser image. Among the whole set of values $(\tilde{\alpha}, \widetilde{\beta})$, formation of $L$ and $\pm C$ polarization states seems to be very probable $[23,29]$.

$$
\begin{aligned}
& L-\Leftrightarrow \delta(\Delta x, \Delta y)=q \pi, \quad q=1,2, \ldots ; \\
& \quad \pm C-\Leftrightarrow \operatorname{tg} \delta(\Delta x, \Delta y)= \\
& =\sin 2\left(\operatorname{arctg}\left\{\left[\left(f_{21}(\Delta x, \Delta y)+f_{22}(\Delta x, \Delta y)\right)^{2} /\left(f_{11}(\Delta x, \Delta y)+f_{12}(\Delta x, \Delta y)\right)^{2}\right] \operatorname{tg} \alpha_{0}\right\}\right)
\end{aligned}
$$

\subsection{Polarization structure of laser fields inherent to PhIL with bulk scattering}

When analyzing the processes of interaction of laser radiation with these PhIL, we have used the method of superposition of the Jones matrix operators (3) for the set of sequentially disposed optically-thin layers:

$\left.\{\Phi\}=\left\{\Phi^{(p)}\right\} \Phi^{(p-1)}\right\} \times \ldots \times\left\{\Phi^{(1)}\right\}$.

Having calculated the set of Jones matrix elements $\phi_{q g}$ for an optically-thick PhIL, one can define analytical expressions (like to (5) - (6)) to find $L$ and $\pm C$ polarization states in the laser image:

$$
\begin{aligned}
& L-\Leftrightarrow \delta^{*}(\Delta x, \Delta y)=q \pi, \quad q=1,2, \ldots ; \\
& \pm C-\Leftrightarrow \operatorname{tg} \delta^{*}(\Delta x, \Delta y)= \\
& =\sin 2\left(\operatorname{arctg}\left\{\left[\left(\phi_{21}(\Delta x, \Delta y)+\phi_{22}(\Delta x, \Delta y)\right)^{2} /\left(\phi_{11}(\Delta x, \Delta y)+\phi_{12}(\Delta x, \Delta y)\right)^{2}\right]\right\} \operatorname{tg} \alpha_{0}\right)
\end{aligned}
$$

Thus, the above analytical consideration (relations (1) to (12)) for various scenarios of transformation of laser radiation by PhIL in all the cases enabled to reveal the principled possibility of formation of polarization-singular states $(\beta=0, \beta= \pm \pi / 4)$ in respective laser images.

In this work, to describe coordinate $(x, y)$ distributions for polarization-singular ( $L, \pm C$ ) states in laser images for all the types of PhIL [23, 29].

$$
\left\{\begin{array}{l}
V_{4}(x, y)=0 \leftrightarrow L(\beta=0) \\
V_{4}(x, y)= \pm 1 \leftrightarrow \pm C(\beta= \pm \pi / 4)
\end{array}\right.
$$




\section{EXPERIMENTAL SETUP FOR POLARIMETRIC INVESTIGATIONS}

Our study of polarization-inhomogeneous laser images inherent to PhIL was performed using the optical scheme of a laser polarimeter (figure 1) $[5,15]$ :

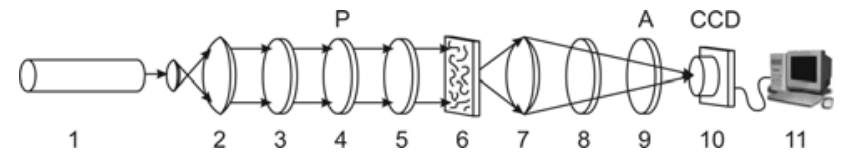

Figure 1. Optical scheme of the polarimeter: 1 - He-Ne laser; 2 collimator; 3, 5, 8 - quarter-wave plates; 4, 9 - polarizer and analyzer, respectively; 6 - object under investigation; 7 - micro-objective; $10-\mathrm{CCD}$ camera; 11 - personal computer.

Illumination was performed using a parallel beam $(\varnothing=$ $\left.10^{4} \mu \mathrm{m}\right)$ from a He-Ne laser $(\lambda=0.6328 \mu \mathrm{m}) 1$. The polarization illuminator (quarter-wave plates 3 and 5 as well as polarizer 4) were used to form various polarization states in the laser beam. Polarization images of PhIL 6 were projected using the micro-objective 7 into the plane of the light-sensitive area $(800$ pix $\times 600$ pix $)$ in CCD-camera 10 . Turning the transmission axis of the analyzer 9 by the angles $\pm 45^{0}$ relatively to the direction of the highest velocity axis for the quarter-wave plate 8 , wee could determine the intensities of right $\left(I_{\otimes}\right)$ and left $\left(I_{\oplus}\right)$ circularly polarized components for each separated pixel of CCD camera 10. It served as a base to calculate coordinate distributions of the fourth parameter in the Stokes vector $\left.V_{4}(m \times n)\right)$ describing the laser image of PhIL, if using the relation:

$V_{4}\left(\begin{array}{ccc}r_{11} & \ldots & r_{1 m} \\ \ldots & \ldots & \ldots \\ r_{n 1} & \ldots & r_{n m}\end{array}\right)=\frac{I_{\otimes}\left(r_{i k}\right)-I_{\oplus}\left(r_{i k}\right)}{I_{\otimes}\left(r_{i k}\right)+I_{\oplus}\left(r_{i k}\right)}$.

The two-dimensional array (14) was scanned along the horizontal direction $x \equiv 1, \ldots, \quad m$ with the step $\Delta x=1$ pix . Within the limits of each local sample $\left(1_{p i x} \times n_{p i x}\right)(k=1,2, \ldots, m)$, we calculated the amount $(N)$ of characteristic values $V_{4}(k)=0,-\left(N_{L}^{(k)}\right)$ and $V_{4}(k)= \pm 1,-\left(N_{ \pm C}^{(k)}\right)$.

Thus, we determined the dependences $N_{L}(x) \equiv\left(N_{L}^{(1)}, \quad N_{L}^{(2)}, \ldots, \quad N_{L}^{(m)}\right)$ and $N_{ \pm C}(x) \equiv\left(N_{ \pm C}{ }^{(1)}, \quad N_{ \pm C}{ }^{(2)}, \quad \ldots, \quad N_{ \pm C}{ }^{(m)}\right)$ for amounts of polarization-singular $L-$ and $\pm C$-points within the limits of a laser image for PhIL.

\section{CRiteria to Estimate Polarization- INHOMOGENEOUS IMAGES OF PHIL}

Distributions $N_{L, \pm C}(x)$ for the amount of polarizationsingular states in laser images of PhIL are characterized with the set of statistical moments of the 1-st to 4-th orders $Z_{j=1,2,3,4}$ calculated using the following relations $[6,7,26$, 31]:

$$
\begin{gathered}
Z_{1}=\frac{1}{M} \sum_{i=1}^{M} \mid N_{L, \pm C}^{(i)}(x), \quad Z_{2}=\sqrt{\frac{1}{M} \sum_{i=1}^{M}\left[N_{L, \pm C}^{(i)}(x)\right]^{2}} \\
Z_{1}=\frac{1}{Z_{2}^{3}} \frac{1}{M} \sum_{i=1}^{M}\left[N_{L, \pm C}^{(i)}(x)\right]^{2}, \quad Z_{4}=\frac{1}{Z_{2}^{4}} \frac{1}{M} \sum_{i=1}^{M}\left[N_{L, \pm C}^{(i)}(x)\right]^{4} .
\end{gathered}
$$

where $N=800 \times 600$ is the amount of pixels in CCD camera 10 (Fig. 1).

Our analysis of the coordinate structure for $N_{L, \pm C}(x)$ distributions was based on the autocorrelation method by using the function $[19,22,27]$ :

$K_{L, \pm C}(\Delta x)=\lim _{m \rightarrow 0} \frac{1}{m} \int_{1}^{m} N_{L, \pm C}(x) N_{L, \pm C}(x-\Delta x) d x$

Here, $(\Delta x=1$ pix $)$ is the step for changing the coordinate $x=1 \div m$.

As correlation parameters that characterize the dependences $K_{L, \pm C}(\Delta x)$, we chose:

- correlation area $S_{L, \pm C}$

$$
S_{L, \pm C}=\int_{1}^{m} K_{L, \pm C}(\Delta x) d x
$$

- correlation moment $Q_{L, \pm C}$ that define the excess for the distribution of values $K_{L, \pm C}(\Delta x)$

$Q_{L, \pm C}=\frac{\sum_{i=1}^{m}\left(K_{L, \pm C}\left(\Delta x_{i}\right)\right)^{4}}{\sum_{i=1}^{m}\left(K_{L, \pm C}\left(\Delta x_{i}\right)\right)^{2}}$.

The fractal analysis of the distributions $N_{L, \pm C}(x)$ was performed using the calculation of logarithmic dependences $\log J\left[N_{L, \pm C}(x)\right]-\log d^{-1}$ for the power spectra $J\left[N_{L, \pm C}(x)\right]$

$$
J=\int_{-\infty}^{+\infty} N_{L, \pm C} \cos 2 \pi v d v
$$

where $v=d^{-1}$ are the spatial frequencies that are determined by geometrical sizes $(d)$ of PhIL structural elements.

The dependences $\log J\left[N_{L, \pm C}(x)\right]-\log d^{-1} \quad$ are approximated using the least-squares method into the curves $\Phi(\eta)$, straight parts of which serve to determine the slope angles $\eta$ and calculate fractal $F$ dimensionalities by using the relations $[6,26]$ :

$F_{L, \pm C}=3-\operatorname{tg} \eta$ 
Classification of coordinate distributions $N_{L, \pm C}(x)$ should be performed using the following criteria [23]:

- they are fractal on the condition of a constant slope angle value $\eta=$ const for 2 to 3 decades of changing sizes $d$;

- they are multi-fractal, if several slope angles $\Phi(\eta)$ are available;

- they are random when any stable slope angles are absent within the whole range of changing sizes $d$.

In the latter case, the distributions $\log J\left[N_{L, \pm C}(x)\right]-\log d^{-1}$ are characterized with the dispersion

$D_{z}=\sqrt{\frac{1}{m} \sum_{i=1}^{m}\left[\log J\left(N_{L, \pm C}\left(x_{i}\right)\right)-\log d^{-1}\right]^{2}}$.

\section{CHARACTERIZATION OF OBJECTS UNDER INVESTIGATION}

Figure 2 illustrates coordinate $(50$ pix $\times 50$ pix $)$ distributions of the fourth parameter for the Stokes vector $V_{4}(m \times n)$ inherent to laser images of PhIL in all the groups.
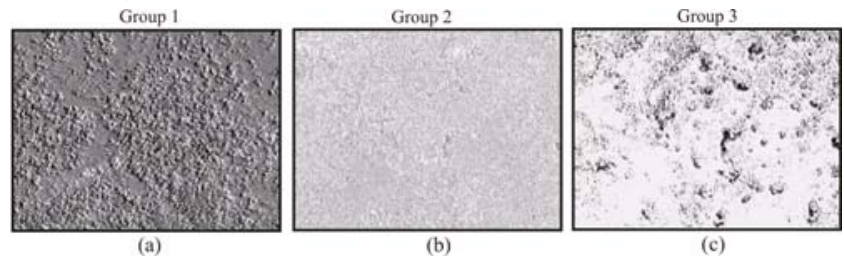

Figure 2. Coordinate distributions $V_{4}(m \times n)$ of laser images inherent to PhIL

Our qualitative analysis of coordinate distributions $V_{4}(m \times n)$ for laser images of PhIL (figure 2) enabled to reveal:

- Practically all the images of the rough surface of skin (figure 2a) are linearly polarized field $V_{4}(m \times n)=0$ (relations (2) and (3)). Availability of a small amount of the parts $V_{4}(m \times n) \neq 0$ polarized otherwise can be related with interferential effects of multiple interaction of coherent waves with adjacent micro-roughnesses.

- The image of the rough skin surface with a subsurface layer of the derma (figure $2 b$ ) is characterized with a developed polarization-inhomogeneous structure formed both by linearly $\left(V_{4}(m \times n)=0\right)$ and elliptically $\left(V_{4}(m \times n) \neq 0\right)$ polarized states, including the circularly $\left(V_{4}(m \times n)=1\right)$ polarized ones (relations (5) to (8)).

- The images of the optically thick layer of skin are characterized with the widest range of changing the azimuth and polarization due to multiple bulk scattering (relations (11) and (12)), $--1 \leq V_{4}(m \times n) \leq 1$.

VI. Statistical, Correlation ANd Fractal ANALyses FOR DISTRIBUTIONS OF POLARIZATION-SINGULAR STATES IN LASER IMAGES OF PHIL

\subsection{L states of laser images}

Summarized in figure 3 is a series of coordinate $\left(V_{4}(m \times n)=0\right)$, quantitative $\left(N_{L}(x)\right), \quad$ autocorrelation
$\left(K_{L}(\Delta m)\right)$ and $\operatorname{logarithmic}\left(\log J_{L}-\log d^{-1}\right)$ distributions for polarization-singular $L$ states in laser images of PhIL.
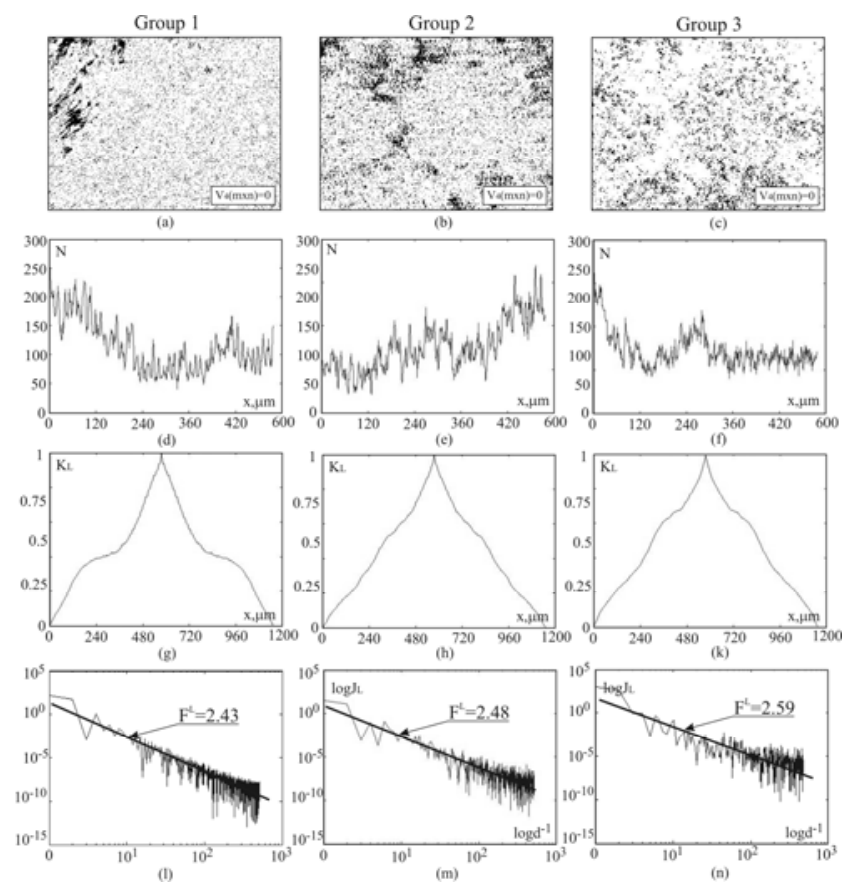

Figure 3. Coordinate $V_{4}(m \times n)=0$ (fragments a, b, c) and quantitative $N_{L}(x)$ distributions (fragments $\mathrm{d}, \mathrm{e}, \mathrm{f}$ ) of $\mathrm{L}$ states in polarization; autocorrelation functions $K_{L}(\Delta x)$ (fragments $\mathrm{g}, \mathrm{h}, \mathrm{k}$ ) and relations $\log J_{L}-\log d^{-1}$ ( fragments $1, \mathrm{~m}, \mathrm{n}$ ) for power spectra $J\left(N_{L}\right)$ of the distribution $N_{L}(x)$ for laser images of the PhILs

Our comparative analysis of the obtained dependences $N_{L}(x)$ for the amount of polarization-singular L states in laser images of $\mathrm{PhIL}$ in all the groups (figures 3d-f) revealed similarity of their statistical, correlation and fractal structures.

For instance, all the $N_{L}(x)$ distributions are close to the equiprobable ones - the condition $Z_{j=3,4}^{L} \prec \prec Z_{j=1,2}^{L}$ is valid for the values of statistical moments (relation (15)). Distinctions between the distributions of $\mathrm{L}$ states in laser images of various PhIL are observed as variations of the 1-st and 2-nd statistical moments - $Z_{1}^{L}=0.62, Z_{2}^{L}=0.13$, $Z_{3}^{L}=0.07, Z_{4}^{L}=0.09 \quad($ group 1$) ; Z_{1}^{L}=0.39, Z_{2}^{L}=0.19$, $Z_{3}^{L}=0.06, \quad Z_{4}^{L}=0.085 \quad\left(\right.$ group 2) and $Z_{1}^{L}=0.19$, $Z_{2}^{L}=0.28, Z_{3}^{L}=0.04, Z_{4}^{L}=0.07$ (group 3).

As seen, for PhIL of the 1-st, 2-nd and 3-rd groups, the mean value $Z_{1}^{L}$ is 1.5 and 3.3 times decreased. And vice versa, the dispersion $Z_{2}^{L}$ is 1.5 and 2.5 times increased. These changes in $N_{L}(x)$ distributions are related with the mechanisms of optically-anisotropic (group 2) and interferential phase modulation (group 3) as well as formation (relations (6) and (10)) of elliptically polarized states in laser images of PhIL. Due to these processes, the total amount of polarization $\mathrm{L}$ states is decreased with simultaneous conservation of their equiprobable disposition in the image plane of various PhIL. 
The investigated statistical structure of $\mathrm{L}$ states for polarization of PhIL laser images is confirmed by a monotonous drop of dependences for autocorrelation functions $K_{L}(\Delta m)$ (relations (16)) of all the distributions $N_{L}(x)$ (fugure $3 \mathrm{~g}, \mathrm{~h}, \mathrm{k}$ ). In this case, values of the correlation area $S$ and correlation moment $Q$ trend to their boundary values $\left(S^{L} \rightarrow 0.33, Q^{L} \rightarrow 0\right.$, relations (17) and (18)) that are characteristic just to equiprobable distributions: $\quad S^{L}=0.26, \quad Q^{L}=0.11 \quad$ (group 1 ); $S^{L}=0.22, Q^{L}=0,14$ (group 2) i $S^{L}=0.19, Q^{L}=0,18$ (group 3).

The performed analysis of logarithmic dependences $\log J_{L}-\log d^{-1}$ (figure 31, m, n) for the power spectra $J\left(N_{ \pm C}\right)$ (relation (19) of the distribution $N_{ \pm C}(x)$ inherent to laser images of the group 1 taken from the group 2, of the group 2 and group 3 revealed a common regularity - the approximating curves are characterized with stable slope angles that are corresponded with increasing by their value fractal dimensionalities (relation (20)): $F^{L}=2.43$ (group $1) ; F^{L}=2.48($ group 2$)$ and $F^{L}=2.59$ (group 3 ).

\section{2. \pm C states of laser images}

Summarized in figure 4 is the series of coordinate $\left(V_{4}(m \times n)=1\right)$, quantitative $\quad\left(N_{ \pm C}(x)\right), \quad$ autocorrelation $\left(K_{ \pm C}(\Delta m)\right)$ and $\operatorname{logarithmic}\left(\log J_{L}-\log d^{-1}\right)$ distributions for polarization-singular $\pm C$-states in laser images of PhIL.

In the laser image of the group $1 \pm C$-states of polarization are absent (figure 4 , left column - fragments a, $\mathrm{b}, \mathrm{d}, 1)$, which corresponds to model conceptions of mechanisms providing transformation of laser radiation by the set of chaotically oriented micro-areas of the rough surface (relations (1) and (2)).

Optical manifestations of the anisotropic layer of collagen fibrils are illustrated with the network of $\pm C$ - points in the laser image (figure 4b). It is seen that the total amount of circularly polarized points (figure $4 \mathrm{c}$ ) is practically one order less than the amount of linearly polarized states (figure $3 \mathrm{~b}, \mathrm{c})$ All the static moments $Z_{j=1,2,3,4}^{ \pm C}=0.48$ that characterize the distribution $N_{ \pm C}(x)$ of the amount of circularly polarized singular states differ from zero: $Z_{1}^{ \pm C}=0.09, \quad Z_{2}^{ \pm C}=0.33, \quad Z_{3}^{ \pm C}=0.48, \quad Z_{4}^{ \pm C}=0.52$. In this case, the values of statistical moments of higher orders $Z_{j=2,3,4}^{ \pm C}$ are commensurable: $Z_{2}^{ \pm C} \approx Z_{3}^{ \pm C} \approx Z_{4}^{ \pm C}$.

This fact is indicative of another, more complex, statistical distribution for the amount of $\pm C$ polarization states as compared with the equiprobable distribution of $\mathrm{L}$ polarization states in laser images of the PhIL (group 2).

The autocorrelation function $K^{ \pm C}(\Delta m)$ of the dependence $N_{ \pm C}(x)$ (figure $4 \mathrm{~h}$ ) rapidly drops with increasing the scanning step $\Delta x$, which shows the random distribution of states with circular polarization in laser images of the ground PhIL. The correlation area $S^{ \pm C}$ and correlation moment $Q^{ \pm C}$ of the distribution $N_{ \pm C}(x)$ as compared with similar correlation parameters of $N_{L}(x)$ distributions (figure $3 \mathrm{~h}$ ) experience changes in inverse proportion: $S^{ \pm C}(\downarrow)=0.16$ and $Q^{ \pm C}(\uparrow)=0.96$.

The random character of the $N_{ \pm C}(x)$ distribution is also confirmed by the absence of a stable value for the slope angle of the approximating curve to the logarithmic dependence $\log J_{ \pm C}-\log d^{-1}$ (figure $4 \mathrm{~m}, \mathrm{n}$ ). In this case, the dispersion value $D^{ \pm C}$ grows up to 2.1 times as compared with the data obtained for logarithmic dependences of power spectra for distributions of the amount of linearly polarized states: $D^{ \pm C}=0.49$.

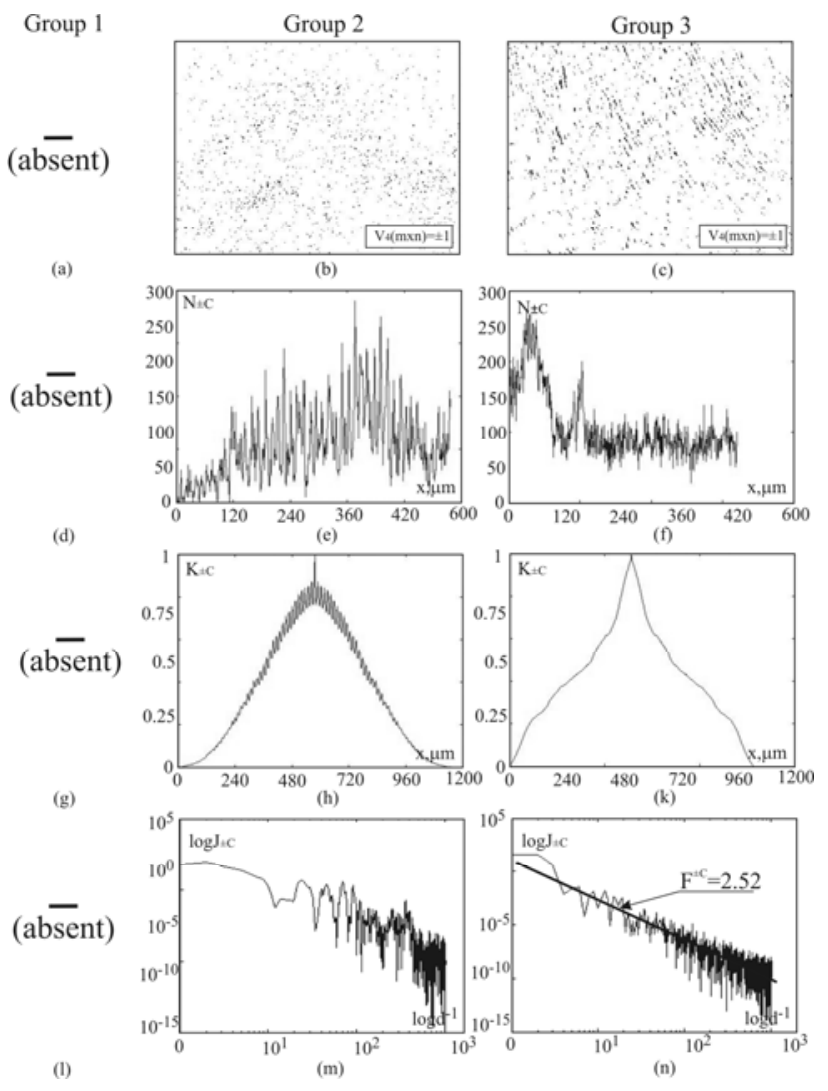

Figure 4. Coordinate $V_{4}(m \times n)=1$ (fragments b, c), and quantitative $N_{L}(x)$ distributions (fragments e, f) of $\pm C$-states in polarization; autocorrelation functions $K_{ \pm C}(\Delta x)$ (fragments $\mathrm{h}, \mathrm{k}$ ), and залежності $\log J_{L}-\log d^{-1} \quad$ (fragments $\left.\mathrm{h}, \mathrm{k}\right)$ for power spectra $J\left(N_{ \pm C}(x)\right)$ (fragments $\mathrm{m}, \mathrm{n}$ ) of the distribution $N_{ \pm C}(x)$ for laser images of PhILs all groups

The phase modulation of multiply scattered laser radiation by the optically thick layer of the skin derma is characterized by the network of $\pm C$-states (figure $4 \mathrm{c}$ ).

The total amount of $L$ - and $\pm C$-states of polarization for the respective laser image is approximately the same (figures $3 \mathrm{f}$ and $4 \mathrm{f}$ ). Like to the case of $N_{L}(x)$ distribution for the amount of linearly polarized states, the $N_{ \pm C}(x)$ distribution is close to the equiprobable one: $Z_{j=3 ; 4}^{ \pm C} \prec \prec Z_{j=1 ; 2}^{ \pm C}$. Differences between statistical moments 
$Z_{j=1,2,3,4}^{L}$ and $Z_{j=1,2,3,4}^{ \pm C}$ are insignificant and lie within 25 to $35 \%: Z_{1}^{ \pm C}=0.14, Z_{2}^{ \pm C}=0.31, Z_{3}^{ \pm C}=0.08, Z_{4}^{ \pm C}=0.14$.

The values of the correlation area $S^{ \pm C}$ and correlation moment $Q^{ \pm C}$ are close to their extremum ones: $S^{ \pm C}=0.225, Q^{ \pm C}=0.17$.

Our analysis of the logarithmic dependences (Fig. 4n) for the power spectra $J\left(N_{ \pm C}\right)$ of the $N_{ \pm C}(x)$ distribution in laser images of the PhIls of group 3 found a stable slope of the approximating curve: $F^{ \pm C}=2.52$. The dispersion value of the distribution $\log J_{ \pm C}-\log d^{-1}$ is 1.7 times grown as compared with the analogous statistical moment that characterizes the dependence $\log J_{L}-\log d^{-1}$ $\left(D^{ \pm C}=0.285\right)$.

\section{POLARIZATION-SingUlar ClASSIFICATION AND DIFFERENTIATION OF OPTICAL PROPERTIES INHERENT TO PHIL}

The statistically averaged (within the limits of groups 1 to 3) values and ranges of changing statistical moments $Z_{j=1 ; 2 ; 3 ; 4}^{L ; \pm C}$, correlation $Q^{L ; \pm C}, S^{L ; \pm C}$ and fractal $F^{L ; \pm C}, D^{L ; \pm C}$ parameters that characterize the $N_{ \pm C}(x)$ dependences for the amount of singular states in laser images of PhIL have been illustrated on Table 1.

TABLE I. STATISTICAL, CORRELATION AND FRACTAL PARAMETERS FOR THE DISTRIBUTION OF THE AMOUNT OF POLARIZATION-SINGULAR STATES IN LASER IMAGES OF PHIL

\begin{tabular}{|c|c|c|}
\hline \multirow{2}{*}{$\begin{array}{c}\text { Parameters } \\
(L-; \pm C-)\end{array}$} & \multicolumn{2}{|c|}{ Group 1} \\
\hline & $L-$ & $\pm C-$ \\
\hline$Z_{1}$ & $0.71 \pm 0.079$ & - \\
\hline$Z_{2}$ & $0.12 \pm 0.034$ & - \\
\hline$Z_{3}$ & $0.05 \pm 0.008$ & - \\
\hline$Z_{4}$ & $0.17 \pm 0.044$ & - \\
\hline$S$ & $0.27 \pm 0.012$ & - \\
\hline$Q$ & $0.08 \pm 0.01$ & - \\
\hline$F$ & $2.43 \pm 0.11$ & - \\
\hline \multirow[t]{2}{*}{$D$} & $0.25 \pm 0.073$ & - \\
\hline & \multicolumn{2}{|c|}{ Group 2} \\
\hline$Z_{1}$ & $0.43 \pm 0.045$ & $0.14 \pm 0.032$ \\
\hline$Z_{2}$ & $0.23 \pm 0.042$ & $0.39 \pm 0.091$ \\
\hline$Z_{3}$ & $0.07 \pm 0.009$ & $0.41 \pm 0.12$ \\
\hline$Z_{4}$ & $0.08 \pm 0.009$ & $0.49 \pm 0.23$ \\
\hline$S$ & $0.24 \pm 0.02$ & $0.18 \pm 0.045$ \\
\hline$Q$ & $0.01 \pm 0.0149$ & $1.01 \pm 0.35$ \\
\hline$F$ & $2.48 \pm 0.012$ & Statistical \\
\hline \multirow[t]{2}{*}{$D$} & $0.27 \pm 0.012$ & $0.49 \pm 0.021$ \\
\hline & \multicolumn{2}{|c|}{ Group 3} \\
\hline$Z_{1}$ & $0.25 \pm 0.035$ & $0.21 \pm 0.041$ \\
\hline$Z_{2}$ & $0.32 \pm 0.045$ & $0.26 \pm 0.034$ \\
\hline$Z_{3}$ & $0.05 \pm 0.007$ & $0.08 \pm 0.01$ \\
\hline$Z_{4}$ & $0.08 \pm 0.03$ & $0.07 \pm 0.05$ \\
\hline$S$ & $0.21 \pm 0.032$ & $0.26 \pm 0.028$ \\
\hline$Q$ & $0.18 \pm 0.034$ & $0.14 \pm 0.019$ \\
\hline
\end{tabular}

\begin{tabular}{|c|c|c|}
\hline$F$ & $2.59 \pm 0.08$ & $2.52 \pm 0.11$ \\
\hline$D$ & $0.22 \pm 0.019$ & $0.25 \pm 0.021$ \\
\hline
\end{tabular}

The performed analysis of results adduced in Table 1 for statistical $\left(Z_{j=1,2,3,4}^{L, \pm C}\right)$, correlation $\left(S^{L, \pm C}, Q^{L, \pm C}\right)$ and fractal $\left(F^{L, \pm C}, D^{L, \pm C}\right)$ parameters has shown:

\section{For PhIL of the group 1:}

- differentiation of optical properties inherent to rough surfaces with different micro-relief parameters is possible when using determination of values for the 2-nd and 4-th statistical moments of the distribution specific to the amount of $\mathrm{L}$ polarization states: with increasing the micro-relief, the value $Z_{2}^{L}$ grows by 1.85 times, while the value $Z_{4}^{L}$ is 2.3 times lowered;

- the dispersion value $D^{L}$ for the distribution of extremes in logarithmic dependences $\log J_{L}-\log d^{-1}$ describing laser images of large-scale (M40) rough surfaces is 2.2 times increased;

- the values of correlation parameters $\left(S^{L, \pm C}, Q^{L, \pm C}\right)$ that characterize the dependences $N_{L}(x)$ for laser images of surfaces with various sizes of micro-roughness (from 1 to $40 \mu \mathrm{m})$ cannot serve as reliable criteria for their differentiation.

2. For PhIL of the group 2:

- the variations of values inherent to all the statistical moments $Z_{j=1,2,3,4}^{ \pm C}$ that characterize the distribution of the amount of $\pm C$-states in the respective laser images were found to be sensitive to changes in the thickness of top and subsurface layers on surfaces ground, $Z_{1}^{ \pm C}$ is 1.6 times decreased; $Z_{2}^{ \pm C}$ is 2.1 times increased; $Z_{3}^{ \pm C}$ increases by 1.8 times and $Z_{4}^{ \pm C}$ is 3.2 times increased;

- there observed are significant differences between the values of correlation areas $S^{ \pm C}$ (1.7 times growth) and correlation moments $Q^{ \pm C}$ (2.9 times drop);

- $N^{ \pm C}$ distributions for the amount of $\pm C$ - states in laser images of all the ground surfaces are statistical - the approximating curves for the dependences $\log J_{ \pm C}-\log d^{-1}$ have no stable slope;

- the dispersion value $D^{ \pm C}$ for the logarithmic dependences of power spectra for the $N^{ \pm C}$ distributions is changed insignificantly (by 1.1 times) and cannot serve as a reliable criterion for differentiation of optical properties of ground surfaces.

3. For PhIL of the group 3:

- there exists a possibility to use statistical moments of the 4-th order that characterize the $N^{L}(x)$ and $N^{ \pm C}(x)$ distributions for differentiation of optically-thick layers with a bulk scattering: with increasing the optical thickness, the differences between $Z_{4}^{L}$ and $Z_{4}^{ \pm C}$ reach 6 and 5 times, respectively;

- weak differences take place between the values of correlation parameters $S^{L, \pm C}, Q^{L, \pm C}: 1.25$ and 1.4 times, respectively;

- $N^{L}(x)$ and $N^{ \pm C}(x)$ distributions are fractal. 


\section{For PhIL of all the groups:}

The possibility to differentiate "group" optical properties of PhIL with surface, subsurface and bulk light scattering is illustrated in Table 2.

TABLE II. The possibility to differentiate "group" optical properties of PhIL with surface, subsurface and bulk light scattering

\begin{tabular}{|c|c|c|}
\hline PhIL & \multicolumn{2}{|c|}{ Groups 1-3 } \\
\hline Parameters & $N_{L}$ & $N_{ \pm C}$ \\
\hline$Z_{1}$ & $\oplus$ & $\oplus$ \\
\hline$Z_{2}$ & $\oplus$ & $\oplus$ \\
\hline$Z_{3}$ & $\otimes$ & $\oplus$ \\
\hline$Z_{4}$ & $\otimes$ & $\oplus$ \\
\hline$S$ & $\otimes$ & $\oplus$ \\
\hline$Q$ & $\otimes$ & $\oplus$ \\
\hline$F$ & $\otimes$ & $\oplus$ \\
\hline$D$ & $\otimes$ & $\oplus$ \\
\hline
\end{tabular}

Note: $\otimes$ - here differentiation is impossible; $\oplus$ - possible

\section{CONCLUSION}

Analyzed in this work are the main physical mechanisms providing formation of polarization singularities in laser images of PhIL with surface, subsurface and bulk light scattering.

Offered are statistical, correlation and fractal parameters for polarization-singular estimating the optical properties inherent to PhIL of all types.

Determined are the ranges for changing the set of criteria that characterize distributions of the amount of polarizationsingular states in laser images, which enabled us to realize both "intergroup" classification and "intragroup" differentiation of optical properties related to PhIL of various types.

\section{REFERENCES}

[1] J.F. de Boer and T.E. Milner, "Review of polarization sensitive optical coherence tomography and Stokes vector determination," J. Biomed. Opt. 7, pp. 359-371 (2002)

[2] J.F. de Boer, T.E. Milner and J.S. Nelson,. Trends in Optics and Photonics (TOPS): Advances in Optical Imaging and Photon Migration (OSA, Washington, DC) (1998).

[3] M.J. Everett, K. Shoenenberger, B.W. Colston and L.B. da Silva, "Birefringence characterization of biological tissue by use of optical coherence tomography," Opt. Lett. 23, p.228-230 (1998).

[4] J. Shuliang, Yu. Wurong, G. Stoica and V. Lihong., Optical fibe based Mueller optical coherence tomography Opt. Lett. 28, p.12061208 (2003).

[5] Alexander G. Ushenko and Vasilii P. Pishak, "Laser Polarimetry of Biological Tissue: Principles and Applications", in Handbook of Coherent-Domain Optical Methods: Biomedical Diagnostics, Environmental and Material Science, vol. I, Valery V. Tuchin, Ed. Boston: Kluwer Academic Publishers, 2004, pp. 93-138.

[6] O. V. Angelsky, A. G. Ushenko, Yu. A. Ushenko, V. P. Pishak, "Statistical and Fractal Structure of Biological Tissue Mueller Matrix Images", in Optical Correlation Techniques and Applications, Oleg V. Angelsky, Ed. Washington: Society of Photo-Optical Instrumentation Engineers , 2007, pp. 213-266.

[7] O.V. Angelsky, A.G. Ushenko, Yu.A. Ushenko, V.P. Pishak, and A.P. Peresunko, "Statistical, Correlation, and Topological Approaches in Diagnostics of the Structure and Physiological State of Birefringent Biological Tissues", in Handbook of Photonics for Biomedical Science, Valery V. Tuchin, Ed. USA: CRC Press, 2010, pp. 21-67.

[8] Alexander G. Ushenko, "Polarization structure of laser scattering fields," Optical Engineering, vol. 34(4), pp. 1088-1093, November 1995.
[9] A.G. Ushenko, "Laser diagnostics of biofractals," Quantum Electronics, vol. 29(12), pp. 1078-1084, December 1999.

[10] O.V. Angel'skii, A.G. Ushenko, A.D. Arkhelyuk, S.B. Ermolenko, D.N. Burkovets, "Structure of matrices for the transformation of laser radiation by biofractals," Quantum Electronics, vol. 29(12), pp. 1074 1077, December 1999.

[11] O.V. Angel'skii, A.G. Ushenko A.D. Arheluk, S.B. Ermolenko, D. N. Burkovets, "Scattering of Laser Radiation by Multifractal Biological Structures," Optics and Spectroscopy, vol. 88(3), pp. 444-448, March 2000.

[12] A.G. Ushenko, "Polarization Structure of Biospeckles and the Depolarization of Laser Radiation," Optics and Spectroscopy, vol 89(4), pp. 597-601, October 2000.

[13] A.G. Ushenko, "Stokes-correlometry of biotissues," Laser Physics, vol. 10(5), pp.1286-1292, May 2000.

[14] A.G. Ushenko, "The Vector Structure of Laser Biospeckle Fields and Polarization Diagnostics of Collagen Skin Structures," Laser Physics, vol. 10(5), pp. 1143-1149, May 2000.

[15] A.G. Ushenko, "Laser polarimetry of polarization-phase statistical moments of the object field of optically anisotropic scattering layers,' Optics and Spectroscopy, vol. 91(2), pp. 313-316, February 2001.

[16] A.G. Ushenko, "Polarization contrast enhancement of images of biological tissues under the conditions of multiple scattering," Optics and Spectroscopy, vol. 91(6), pp. 937-940, August 2001.

[17] A.G. Ushenko, "Laser probing of biological tissues and the polarization selection of their images," Optics and Spectroscopy, vol. 91(6), pp.932-936, August 2001.

[18] A.G. Ushenko, "Correlation processing and wavelet analysis of polarization images of biological tissues," Optics and Spectroscopy, vol. 91(5), pp.773-778, June 2002.

[19] A.G. Ushenko, "Polarization correlometry of angular structure in the microrelief pattern or rough surfaces," Optics and spectroscopy, vol. 92(2), pp. 227-229, June 2002.

[20] O.V. Angelsky, A.G. Ushenko, Ye.G. Ushenko, “2-D Stokes Polarimetry of Biospeckle Tissues Images in Pre-Clinic Diagnostics of Their Pre-Cancer States," Journal of Holography and Speckle, vol. 2(1), pp. 26-33, April 2005.

[21] Oleg V. Angelsky, Alexander G. Ushenko, and Yevheniya G Ushenko, "Complex degree of mutual polarization of biological tissue coherent images for the diagnostics of their physiological state," J. Biomed. Opt., vol. 10(6), Article ID 060502, November 2005.

[22] O. V. Angelsky, A. G. Ushenko, and Ye. G. Ushenko, "Investigation of the correlation structure of biological tissue polarization images during the diagnostics of their oncological changes," Physics in Medicine and Biology, vol. 50(20), pp. 4811-4822, September 2005.

[23] Oleg V. Angelsky, Alexander G. Ushenko, Yevheniya G. Ushenko, Yuriy Y. Tomka, "Polarization singularities of biological tissues images," J. Biomed. Opt., vol. 11(5), Article ID 054030, SeptemberOctober 2006.

[24] O.G. Ushenko, S.G. Guminetsky, A.V. Motrich, "Optical properties of urine, blood plasma and pulmonary condensate of the patients with pulmovnary form of tuberculosis," Photoelectronics, vol.16, pp. 133139, June 2007.

[25] S.H. Guminetskiy, O.G. Ushenko, I.P. Polyanskiy, A.V. Motrych F.V. Grynchuk, "The optical method for investigation of the peritonitis progressing process," Proceedings of the SPIE, vol. 7008, Article ID 700827, April 2008.

[26] Alexander Ushenko, Sergej Yermolenko, Alexander Prydij, Stepan Guminetsky, Ion Gruia, Ovidiu Toma, Konstantin Vladychenko, "Statistical and fractal approaches in laser polarimetry diagnostics of the cancer prostate tissues," Proceedings of the SPIE, vol. 7008, Article ID 70082C, April 2008.

[27] A.G. Ushenko, A.I. Fediv, Yu.F. Marchuk, "Correlation and fractal structure of Jones matrices of human bile secret," Proceedings of the SPIE, vol. 7368, Article ID 73681Q, July 2009.

[28] A.G. Ushenko, Yu.Ya. Tomka, V.I. Istratiy, "Polarization selection of two-dimensional phase-inhomogeneous birefringence images of biotissues," Proceedings of the SPIE, vol. 7388, Article ID 73881L December 2009.

[29] A.G. Ushenko, A.I. Fediv, Yu.F. Marchuk, "Singular structure of polarization images of bile secret in diagnostics of human physiological state," Proceedings of the SPIE, vol. 7368, Article ID 73681 S, July 2009

[30] S.B. Yermolenko, A.G. Ushenko, P. Ivashko, "Spectropolarimetry of cancer change of biotissues," Proceedings of the SPIE, vol. 7388, Article ID 73881D, December 2009.

[31] A.G. Ushenko, I. Z.Misevich, V. Istratiy, I. Bachyns'ka, A. P. Peresunko, Omar Kamal Numan, and T. G. Moiysuk, "Evolution of Statistic Moments of 2D-Distributions of Biological Liquid Crystal Net Mueller Matrix Elements in the Process of Their Birefringent 
Structure Changes," Advances in Optical Technologies, vol. 2010, Article ID 423145, March 2010.

[32] Yu. A. Ushenko, A. P. Peresunko, and Bozan Adel Baku, "A New Method of Mueller-Matrix Diagnostics and Differentiation of Early Oncological Changes of the Skin Derma," Advances in Optical Technologies, vol. 2010, Article ID 952423, March 2010

[33] Oleg V. Angelsky and Yuriy A. Ushenko, "The Degree of Mutual Anisotropy of Biological Liquid Crystals Net during the Diagnostics of Human Tissues Birefringence," Advances in Optical Technologies, vol. 2010, Article ID 321275, March 2010.

[34] Oleg V. Angelsky, Yuriy A. Ushenko, Alexander V. Dubolazov, and Olha Yu. Telenha, "The Interconnection between the Coordinate Distribution of Mueller-Matrixes Images Characteristic Values of Biological Liquid Crystals Net and the Pathological Changes of Human Tissues," Advances in Optical Technologies, vol. 2010, Article ID 130659, March 2010.

[35] I.Z. Misevitch, Yu.O. Ushenko, O.G. Pridiy, A.V.Motrich, Yu.Ya. Tomka, O.V. Dubolazov, "Investigation of singularities inherent to Mueller matrix images of biological crystals: diagnostics of their birefringent structure," Semicond. Physics, Quantum Electronics\&Optoelectronics, 12(4), 379-390, 2009.

[36] Yu.O. Ushenko, Yu.Ya. Tomka, O.G. Pridiy, A.V.Motrich, O.V. Dubolazov, I.Z. Misevitch, V.V. Istratiy, "Wavelet analysis for Mueller matrix images of biological crystal networks," Semicond. Physics, Quantum Electronics\&Optoelectronics, 12(4), 391-398, 2009.

[37] Yu.O. Ushenko, V.V. Istratiy, V.O. Balanets'ka, D.O. Kvasniyk, V.T. Bachinsky, O.I. Olar, "Phase maps for networks of polycrystalline human biological liquids: Statistical and fractal analyses," Semicond. Physics, Quantum Electronics\&Optoelectronics, vol. 13(3), 240-247, 2010.

[38] Yu.O. Ushenko, I.Z. Misevich, A.P. Angelsky, V.T. Bachinsky, O.Yu. Telen'ga, O.I. Olar, "Polarization-singular structure in laser images of phase-inhomogeneous layers to diagnose and classify their optical properties," Semicond. Physics, Quantum Electronics\&Optoelectronics, vol. 13(3), 248-258, 2010.

[39] V.T. Bachinsky, Yu.O. Ushenko, Yu.Ya. Tomka, O.V. Dubolazov, V.O. Balanets'ka, A.V. Karachevtsev, "Wavelet analysis for polarization maps of networks formed by liquid biological crystals in blood plasma: statistical and fractal approaches," Semicond. Physics, Quantum Electronics\&Optoelectronics, vol. 13(3), 189-201, 2010.
[40] Yu. Ushenko; O. Telenha; V. Balanetskyaya, "Laser metrology of biological liquid crystals singular structure," Proceedings of the SPIE vol. 7821, 78210Z, March 2010 .

[41] Yu. Ya. Tomka, Yu. A. Ushenko, A. O. Karachevtcev, "Laser metrology of biological crystals singular structure," Proceedings of the SPIE vol. 7388, 73881J, December 2009

[42] Yu. A. Ushenko, V. T. Bachinskyi, I. V. Bachinskaya, "Evolution of power spectra of biological tissues Mueller matrix images in the process of changing their birefringent structure," Proceedings of the SPIE vol. 7388, 73881M, December 2009.

[43] Yu. A. Ushenko, P. T. Popovitch, S. V. Godnyuk, "Evolution of statistic moments of 2D distributions of biological tissues Mueller matrix elements in the process of their birefringent structure changes," Proceedings of the SPIE vol. 7388, 73881N, December 2009.

[44] Yuriy A. Ushenko, Yuriy Ya. Tomka, Alexander Dubolazov, "The degree of mutual correlation of coordinate distributions of Mueller matrix elements biological tissues and diagnostics of their physiological state," Proceeding Sensor 2009, pp. 347-351, July 2009.

[45] A.G. Ushenko, I.Z. Misevitch, Yu.A. Ushenko and A.G. Pridiy, "Laser metrology of organic crystals singular structure," Proceeding ISMTII, pp. 262-266, July 2009

[46] A.G. Ushenko, Yu.A. Ushenko, I.Z. Misevitch and A.I. Dubolazov, V.I. Istratyy, "Polarization metrology of Mueller matrices images of phase-inhomogeneous," Proceeding ISMTII, pp. 267-271, July 2009.

[47] A.G. Ushenko, I.Z. Misevitch ,Yu.A. Ushenko and A.G. Pridiy, "Mueller-matrixes tomography of biological crystals network," Proceeding ISMTII, pp. 272-275, July 2009.

[48] S.B. Yermolenko, M.P. Gorsky, Y. A. Ushenko, A.G. Pridiy, "Polarization interferometry of singular structure of organic crystal polarization properties," Proceeding FRINGE 2009, pp. 221-224, September 2009

[49] Sergey B. Yermolenko, Yurij A. Ushenko, Vadim I. Istratyy, Pavlo V. Ivashko, "Polarization selection of two-dimensional phaseinhomogeneous pathologically changed biological tissue images," Proceedings of the SPIE vol. 7371, 73711I, May 2009.

[50] Sergey Yermolenko, Yurij Ushenko, Alexander Prydiy, Stepan Guminetski, Ion Gruia, "Spectropolarimetry in singular structure biotissue images for diagnostics of their pathological changes," Proceedings of the SPIE vol. 7371, 73711M, May 2009.

[51] Yu. A. Ushenko, "Skin as a transformer of the polarization structure of laser radiation," Optics and Spectroscopy vol. 93(3), pp. 321-325, September 2002. 